\title{
Implementation of the University of Louisville COVID-19 Biobank: Experiences from the Center of Excellence for Research in Infectious Diseases (CERID)
}

\author{
Dawn Balcom ${ }^{1}$; Leslie A Wolf ${ }^{1}$; Subathra Marimuthu ${ }^{1}$; William Johnson ${ }^{1}$; Danial Malik ${ }^{1}$; Chet Dhakal ${ }^{1}$; Pavani Nathala ${ }^{1}$; Mohammed Abbas ${ }^{1}$; \\ Sahaj Hardeep Singh ${ }^{1}$; Rafik Elbeblawy ${ }^{1}$; Fnu Deepti ${ }^{1}$; Ahmed Ali ${ }^{1}$; Evelyn Exposito Gonzalez ${ }^{1}$; Basel Haddad ${ }^{1}$; Ruth Carrico $^{1 *}$ \\ ${ }^{1}$ Center of Excellence for Research in Infectious Diseases, Division of Infectious Diseases, University of Louisville, Louisville, KY, USA \\ *rmcarr04@louisville.edu
}

Recommended Citation: Balcom D, Wolf LA, Marimuthu S, et al. Implementation of the University of Louisville COVID-19 Biobank: Experiences from the Center of Excellence for Research in Infectious Diseases (CERID). Univ Louisville J Respir Infect 2020; 4(1):Article 71. doi: 10.18297/jri/vol4/iss1/71.

\begin{abstract}
The limited availability of biological samples from patients testing positive for SARS-CoV-2 for future research was quickly identified at the onset of the COVID-19 pandemic. In response to this need, a COVID-19 biobank was initiated by the University of Louisville Division of Infectious Diseases, Center of Excellence for Research in Infectious Diseases (CERID). The COVID-19 biobank contains waste samples obtained from patients hospitalized with COVID-19 in
\end{abstract}

any of the nine Louisville, Kentucky, area hospitals during the timeframe of April 13 to September 20, 2020. The COVID19 biobank stores approximately 1,623 waste samples with 2,127 aliquots for distribution. All samples are linked to clinical data. The methods described in this paper are intended for use as a guide for other research institutions interested in developing a COVID-19 biobank.

\section{Introduction}

The ability of the novel SARS-CoV-2 virus to produce a broad spectrum of clinical presentations in patients has prompted significant research interest across multiple healthcare disciplines. In March 2020, the University of Louisville Division of Infectious Diseases Center of Excellence for Research in Infectious Diseases (CERID) initiated a COVID-19 surveillance program enabling testing of patients seen in any of the nine Louisville and two southern Indiana area hospitals. This surveillance work resulted in the testing of more than 6000 respiratory samples for SARS-CoV-2 of which 1,026 tested positive for the virus.[1] The development of the surveillance process was used as a guide to develop the COVID-19 biobank process (IRB\# 050556).[2] These positive specimens were retained in the existing CERID Biobank focused on emerging pathogens. This original biobank was implemented in 2005 focusing on discovery of new and emerging pathogens and biomarkers. This biobank currently houses more than 70,000 human samples all linked to clinical data maintained in a webbased secure REDCap database.[4] To enable rapid access to specimen by multiple disciplines interested in this novel virus, a new branch of the biobank was ini- tiated. The development of the COVID-19 biobank included creating new methods to identify patients hospitalized with COVID-19, obtain, store, release, and inventory waste specimens and associate these specimens with clinical data.[3] For this to occur, the new biobank required development of additional organizational capacity that could provide a foundation for anticipated growth and increased interaction with clinicians and basic scientists. The following information outlines the implementation approach for the CERID COVID-19 Biobank.

\section{Objectives}

The objectives of this program review are to describe the steps involved in initiating a COVID-19 biobank process including: 1) methods to identify hospitalized patients with a COVID-19 positive test result and/or ground glass opacities on chest CT; 2) processes for obtaining waste specimen from patients identified with COVID-19; 3) logistical procedures to catalogue and label specimen for storage, location, and retrieval; 4) database development and clinical data entry; 5) quality assurance review; and 6) description of organizational capacity necessary to successfully operate and 
manage a COVID-19 biobank.

\section{Methodology}

The University of Louisville Division of Infectious Diseases implemented a biobank of specimen in 2005 in response to the growing concerns with multidrug resistance and emerging pathogens. The focus of that biobank has been the capture of specimen with a link to clinical data of the patient in an effort to support research in both the clinical care and basic sciences. That current biobank contains more 70,000-patient specimen including blood, urine, tissue and respiratory samples, all linked to clinical data maintained and managed using HIPAA-compliant electronic capture data tools hosted at the University of Louisville. REDCap (Research Electronic Data Capture) is a secure, web-based software platform designed to support research studies.[4] The process of expanding the existing biobank and implementing a new component focusing on COVID-19 branch required development of a comprehensive process utilizing an array of resources. The following steps were undertaken by the CERID team members in development of this new biobank component: 1) development of a new database and corresponding data dictionary focusing on clinical patient data corresponding to the collected waste samples; 2) creation of an identification coding process for each sample; 3) procedures for specimen collection and transportation from each of the nine Louisville area hospitals to the biobank site; 4) development of a data collection manual and data entry process; 5) systems for processing samples for placement in the biobank; 6) procedures for cataloging the samples for location and retrieval; 7) procedures for sample identification and labeling; 8) procedures for storage of samples in the biobank; 9) development of a process for researcher and industry access to biobank sample menu and request process; and 10) development and implementation of quality assurance procedures and metrics.

\section{Specimen database development}

Members of the CERID Biostatistics and Data Management Units created the COVID-19 REDCap database to support the biobank objectives of maintaining, analyzing, and sharing COVID-19 patient samples and related clinical data with the research community. This required that the database development team have the ability to provide basic patient information outlined in the Institutional Review Board (IRB) approval. Data elements included patient demographics, history and physical examination, laboratory and radiologic findings, medication history, and clinical course of illness. Development of the database required parallel development of a data dictionary that described each data element along with acceptable ranges and rules for data entry. For example, formats for date of birth, hospital codes, and general laboratory result ranges were included and drop-down boxes development as a means of addressing preventable data entry error. The database was then pilot tested using paper data collection tools that assisted with rapid development without wasted effort correcting or changing major database approaches or elements. The end result is a biobank database able to provide an efficient mechanism to grow, maintain and monitor the SARS-CoV-2 sample inventory, and track and make available samples to researchers worldwide.

\section{Creation of the sample identification code}

To differentiate the samples entering the COVID-19 biobank, a unique subject identification code and sample labels were created. The abbreviation of "COD" was used to designate the COVID-19 project. Each participating hospital was assigned a hospital code to identify the origination of the sample and the clinical data. Each sample was also provided a sample number that would allow placement of sample aliquots in similar locations for ease in retrieval. An example of the identification code for these samples is "COD-010001". Using this alphanumerical system allowed for additional hospital sites to be added and enabled up to one thousand samples from each hospital to be stored in the COVID-19 biobank. The Marketing Unit then created a prototype of the sticker using that specimen labeling convention for consistency in appearance.

\section{Specimen collection and transport from hospitals to the} biobank

The initial IRB approval allowed for collection of waste samples from hospitalized patients diagnosed with COVID-19. Individual procedures for sample collection were developed at each hospital recognizing that procedures in facility laboratories reflected their local practices. Initial meetings were held with laboratory managers to outline the goals of the biobank, processes for identify patients with COVID-19, types of waste specimen requested, procedures for identifying those samples, and a location for specimen to be held awaiting pickup by CERID researchers. Specimen were transported by CERID researchers to the biobank using temperature-controlled transport containers and immediately placed in a designated refrigerator upon arrival to the biobank.

Procedures varied widely among participating hospital laboratories. For example, one hospital chose to have the Infection Preventionist provide a list of patient names and medical record numbers (MRNs) so they could develop lists to provide to the hospital laboratory. Another hospital assigned that responsibility to one of the infectious diseases physicians, while another authorized access to screening reports via their electronic medical record system. A major goal of this aspect of work involved identification of a process that 

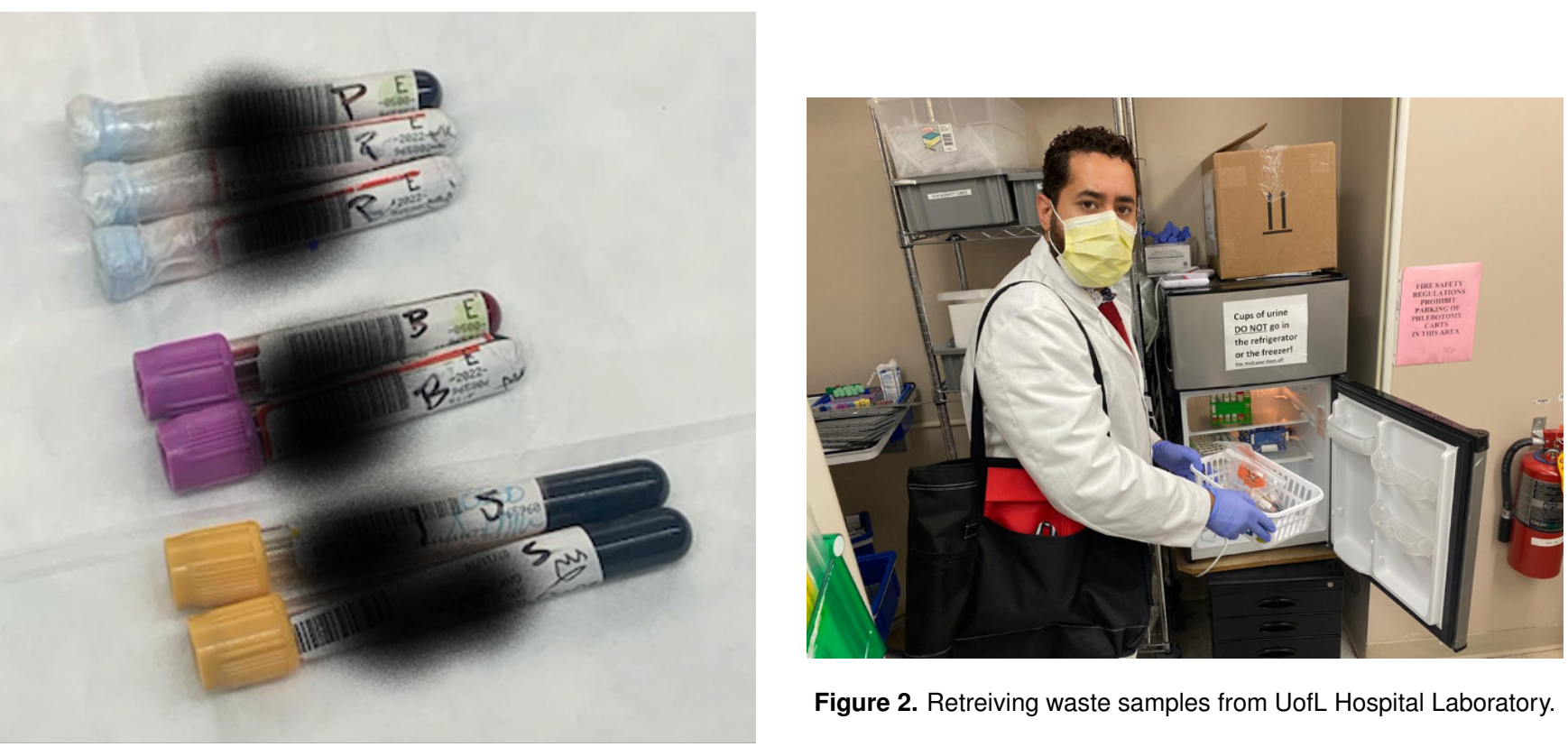

Figure 2. Retreiving waste samples from UofL Hospital Laboratory.

Figure 1. ID label and sample type.

was sustainable for both the hospital and researcher while also minimizing disruption to hospital personnel work and workflow.

After identifying new patients that met the biobank inclusion criteria, a report was generated that included patient name, date of birth and medical record numbers (MRN). The list was cumulative with patients being removed from the list after discharge. This enabled a process to add waste specimen and clinical data throughout the course of hospitalization. Waste samples for the COVID-19 biobank include whole blood, serum, plasma, urine, stool, nasopharyngeal swabs, oropharyngeal swabs, sputum, bronchoalveolar lavage, pleural fluid, cerebrospinal fluid and tissue. Each waste sample was already labeled with patient information according to the individual hospital procedures (Figure 1). Laboratory personnel at the individual hospital placed patient samples into a biohazard bag then placed in a designated refrigerator in the laboratory area. CERID team had placed a basket clearly labelled as COD into the designated refrigerator for CERID researchers to pick up (Figure 2).

Upon arrival to the biobank, a color-coded label was applied with the unique identifier for each hospital and the sequential specimen number as previously described. Each hospital had been designated a specific color. When the samples were logged in to the biobank, the hospital-specific forms were retrieved from premade color specific folders and the corresponding Biobank Screening Log form and a set of COVID-19 Subject ID regular and Cryogenic labels (Figure 3) were used to document receipt of the specimen and provide labels for the tubes. The COVID-19 Biobank Screening Log was completed with patient details (name, date of birth, medical record number). The waste sample details (type, date of collection, accession number assigned by the providing hospital) were then entered onto this form. A final check was done to ensure all samples collected were documented on the screening log. A subject COD ID label was placed on the sticker on the front of the colored file folder, on the COVID19 Biobank Screening Log form and on the biohazard bag. Cryogenic labels were placed inside the biohazard bag. At this point, all information documented on the COVID-19 Biobank Screening Log was handwritten and in ink.

The biohazard bag containing the waste samples and the Cryogenic labels were then placed in a designated labeled basket in the biobank refrigerator for laboratory personnel to process (Figure 4). For patients that were previously entered into REDCap, the COVID-19 Biobank Screening Log was updated with the new sample details (type of sample, date of collection, accession number). New Cryogenic labels with the same Subject ID of the patient were printed and placed in the biohazard bag. The CERID researcher was then responsible for data entry for all specimen received and placement of the file folder(s) in a designated area for quality team review. 

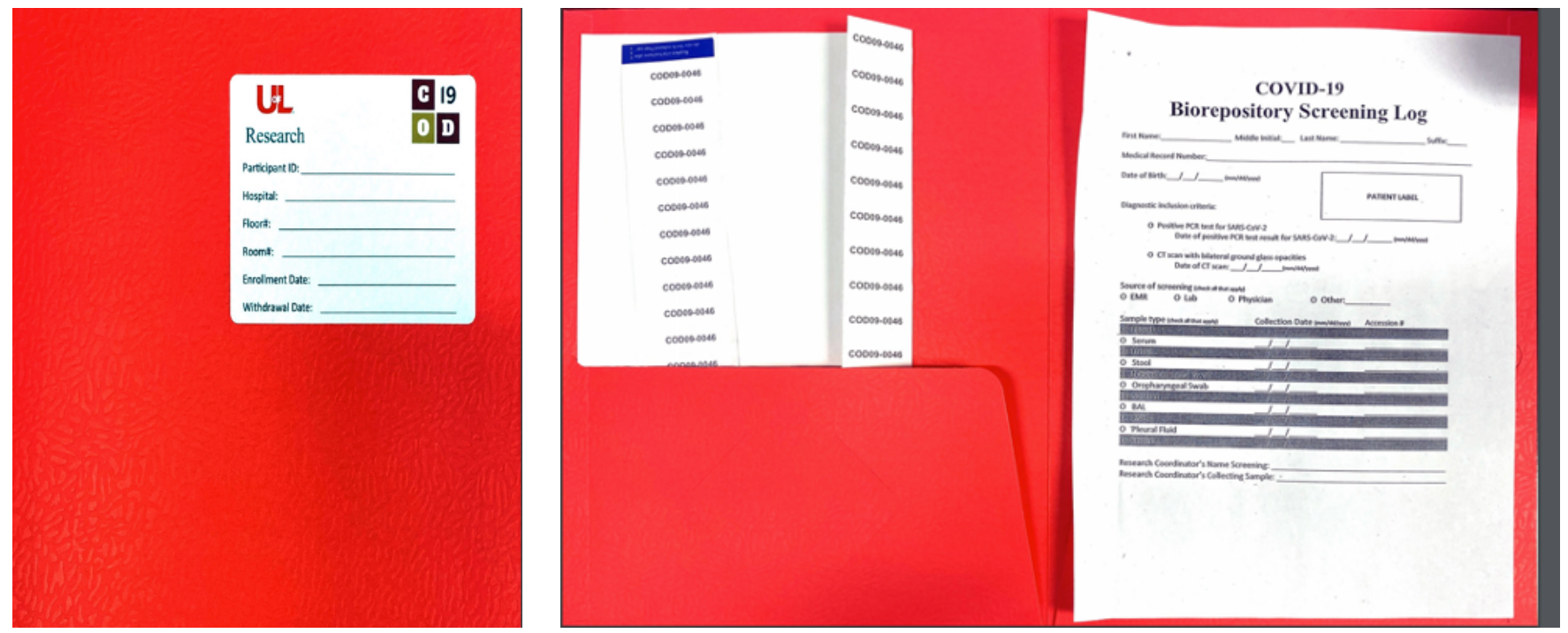

Figure 3. UofL Hospital folder, labels, and biobank screening log.

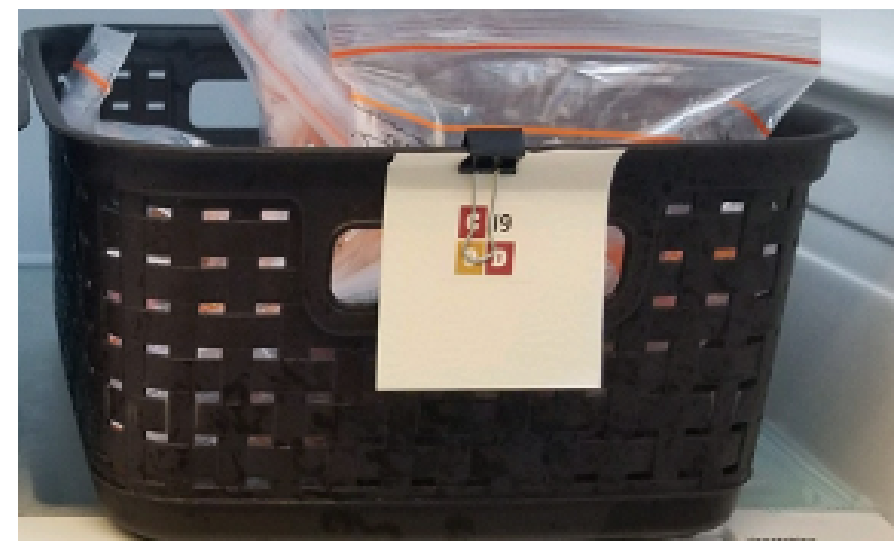

Figure 4. Designated basket for COVID-19 waste samples. 


\begin{tabular}{|c|c|c|c|}
\hline \multicolumn{4}{|l|}{ COVID-19 Retrospective } \\
\hline Actions: 그 Download PDF of instrument(s) & \multicolumn{3}{|c|}{ IEl VIDEO: Basic data entry. } \\
\hline \multicolumn{4}{|l|}{ - Initial Data Entry } \\
\hline & \multicolumn{2}{|r|}{ Assign record to a Data Access Group? } & - select a group - $\checkmark$ \\
\hline \multicolumn{4}{|l|}{ (9) Adding new Record ID 978} \\
\hline Record ID & \multicolumn{2}{|r|}{978} & \\
\hline \multicolumn{4}{|l|}{ Principal Investigator: Julio Ramirez, MD } \\
\hline $\begin{array}{l}\text { Screening Date } \\
\text { * must provide value }\end{array}$ & 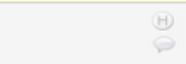 & 07-14-2020 교 Today M-D-Y & \\
\hline $\begin{array}{l}\text { Hospital/Facility/Clinic: } \\
\text { * must provide value }\end{array}$ & $\stackrel{(H)}{\ominus}$ & University of Louisville & $\checkmark$ \\
\hline $\begin{array}{l}\text { First Name: } \\
\text { * must provide value }\end{array}$ & 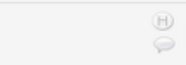 & Roxanna & \\
\hline Middle Initial: & $\stackrel{(H)}{\ominus}$ & & \\
\hline $\begin{array}{l}\text { Last Name: } \\
\text { - must provide value }\end{array}$ & 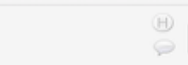 & Smith & \\
\hline Suffix: & 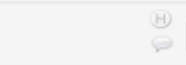 & & \\
\hline $\begin{array}{l}\text { Date of Birth } \\
\text { * must provide value }\end{array}$ & $\stackrel{(H)}{\ominus}$ & 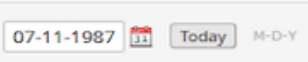 & \\
\hline $\begin{array}{l}\text { Medical Record Number: } \\
\text { - muat provide value }\end{array}$ & $\Theta$ & T003077869 & \\
\hline
\end{tabular}

Figure 5. Initial data entry form.

\section{COVID-19 Retrospective}

Actions: E Download PDF of instrument(s) $\nabla$ VIDEO: Basic data entry.

\section{Biorepository Information}

Data Access Group: [No Assignment] ?

\begin{tabular}{|l|l|l|l|l|}
\hline Editing existing Record ID 935 & \\
\hline Record ID & Sample types collected for biorepository & \multicolumn{1}{|c|}{ Today } \\
\hline Blood (check all that apply) \\
(Additional samples should be approximately one week apart)
\end{tabular}

Figure 6. Biobank information. 


\section{REDCap data entry}

Initial data entry into REDCap occurred at the biobank when the implementation team arrived to drop off samples obtained from the hospitals for processing. There were two sections in the REDCap database to be completed at the biobank; 1) initial patient data, and 2) Biobank Information Sections (Figure 5 and Figure 6). Completing these sections at the biobank provided the laboratory personnel all required information necessary to promptly process the samples for storage. The information in these two sections contain information regarding the who, what, when, and where regarding both the patient and sample history. Beyond allowing the CERID team to begin to build a data history for each sample, this process generated a REDCap unique data identification code. This code is an alphanumeric marker for each sample that allowed researchers to deidentify samples, track samples for inventory purposes, and for the team to securely attach clinical data to the sample record in the future. After the Initial Data and Biobank sections of the database were completed, the samples were placed in a refrigerator for the laboratory personnel to process.

Standard data elements for each patient entered into REDCap included the following data points:

1. Cycle threshold $(\mathrm{Ct})$ value from $\mathrm{PCR}$ assay for SARS-CoV-2

2. Hospitalization date and admitting diagnosis

3. Patient health history

- Signs \& symptoms within 24 hours from onset of symptoms

- Past social history

- Psychiatric history

- Past medical history

- History of Pulmonary and Respiratory Infections/Disease

- HIV history

- Risk factors for cardiovascular events

- Chronically used medications prior to onset of symptoms

4. Maternity-specific data

5. Physical examination and laboratory findings

6. ICU course and ICU lab data

7. Radiological and CT findings

8. Microbiological studies

9. Management and therapy of COVID-19
10. Clinical course during hospitalization

11. Cardiovascular events

12. Clinical outcomes

13. Notes (for comments)

Processing of the samples for placement in biobank

The processing of waste samples for storage in the biobank began immediately after sample arrival, labeling and specimen data entry. The biobank laboratory staff completed the following steps: a) created a daily paper biobank inventory log used for tracking all received sample types, $b$ ) transferred this information into the biobank's electronic Microsoft Excel file, c) applied COD ID label(s) to the designated Eppendorf CRYO tube(s) and documented the sample type (e.g. blood, plasma, serum, urine, nasopharyngeal swabs), volume and location (e.g., freezer, shelf, drawer) of the sample(s) into REDCap database. This information was used to easily identify sample type and location for future retrieval.

The processing of the COVID-19 sample for storage in the biobank occurred in a biosafety level 2 laboratory (BSL-2) in a biosafety cabinet (BSC). This bio-level certification provided the required safeguards when working with bio-specimen agents associated with human diseases. These sample processing procedures requires the participation of two trained laboratory technicians to complete the work safely and accurately. The processing team consists of two laboratory technicians, one who completed the bio-processing work under the BSC and a second technician who worked in parallel to handle the data collection and inventory documentation of the process. This lab work required the following supplies; a single channel repeater pipette, disposable transfer pipettes and $1 \mathrm{~mL}$ pipette, repeater pipette tip, micro pipette tips, $1.7 \mathrm{ml}$ CRYO tubes, Falcon tubes, PIPES buffer, preprinted CRYO stickers with subject ID, CRYO boxes / freezer storage boxes for sample storage. When laboratory technicians processed the biological samples in the BSC, they follow specific laboratory safety guidelines and protocols based on the type of biological sample type being processed at the time. These specific process protocols are described in the following paragraphs.

\section{Blood and respiratory samples}

Two laboratory technicians were required to process daily samples. One laboratory technician began the process by donning the appropriate PPE (gown, gloves, and facemask) prior to beginning to work with the COVID-19 biospecimen in the BSC. When processing respiratory and blood samples laboratory personnel started by opening the sample biohazard bag and placing the hospitals collection tube(s) in a sample tube 


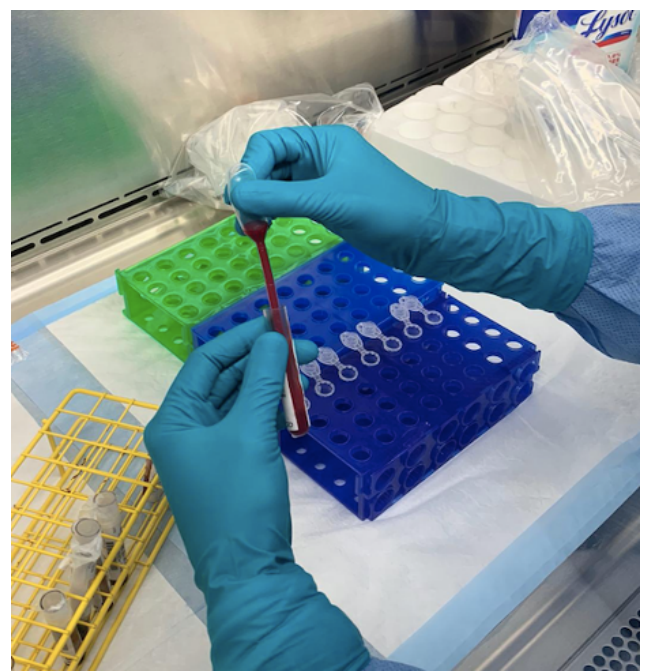

Figure 7. Aliquoting blood into CRYO tube.

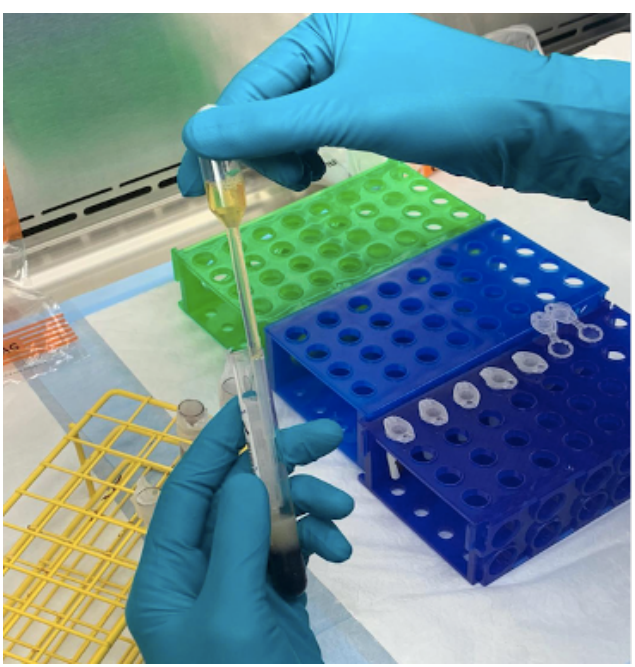

Figure 8. Aliquoting plasma into CRYO tube. rack. Next, the technician mixed the sample in the first collection tube to ensure the uniformity of the specimen in the tube prior to aliquoting it. The technician then opened the first collection tube and began to pipette the sample from the collection tube into a minus eighty degree rated $1.7 \mathrm{~mL}$ CRYO tubes(s) (Figure 7). When aliquoting the sample, technician pipetted an equal volume of $0.5 \mathrm{~mL}$ into each CRYO tube and securely tightened the cap on each CRYO tube and places the tube into a rack. This transfer process continued until the entire collection tube's volume has been pipetted into CRYO tube(s). The technician continued this process until all the respiratory and blood sample collection tube(s) from all subjects of interest in this study for the current day had been transferred into the CRYO tubes and racked.

\section{Urine samples}

Laboratory technician donned the required PPE (gown, gloves, and facemask) prior to beginning the process. Laboratory technician opened the sample biohazard bag in the BSC and placed the hospitals collection cup next to the sample tube rack. The personnel then added $0.250 \mathrm{ml}$ of PIPES buffer to a new Falcon tube location on the tube rack in the hood. Next, the technician opened the urine sample and uses a transfer pipette to add $4.75 \mathrm{~mL}$ of the urine sample to the buffer in the Falcon tube. The Falcon tube was then sealed, and the tube was continuously inverted (a minimum of ten times) until it is well mixed. Once the tube is mixed, the urine sample was aliquoted into $0.5 \mathrm{~mL}$ in each CRYO rated $1.7 \mathrm{~mL}$ Eppendorf tubes and capped on the completed tube rack. This urine sample transfer process to the CRYO tubes continued until the Falcon tub is empty. The technician continued this process until all the urine collections from the current day had been transferred into the CRYO tubes and placed on completed rack.

\section{Stool samples}

In processing the COVID-19 stool sample(s) once the PPE was donned, laboratory technicians began by retrieving the stool sample from the biohazard bag and inspected the sample to identify if any Cary Blair (C.Blair) solution would be required to increase the viscosity of the sample being transferred from the collection cup into the CRYO tube(s). If the sample contained the needed fluidity, then the sample was transferred directly into the CRYO tube(s). If the sample was solid in consistency, then laboratory technician would pipette a small volume of C.Blair solution into the collection cup and mix the sample to loosen it to a level where transfer is achievable. Once the required volume had been transferred the CRYO tube was securely closed and placed on completed rack. The technician continues this process until all the stool collection samples from the current delivery had been transferred into the CRYO tubes and placed on completed rack.

\section{Plasma samples}

After donning proper PPE, laboratory technician begins the process by opening the biohazard bag and confirming the waste sample as plasma. This identification is done by inspecting the label on the collection tube for plasma designation, referring to the laminated informational guide for identifying sample types based on the lid color of the collection tube or inspecting the contents of the tube to confirm if there is a distinctly separated blood clot resting in the bottom of the tube. The laboratory personnel then aliquoted $0.5 \mathrm{~mL}$ of the plasma sample off the supernatant layer and pipettes it into a CRYO tube (Figure 8). Once the sample trans- 


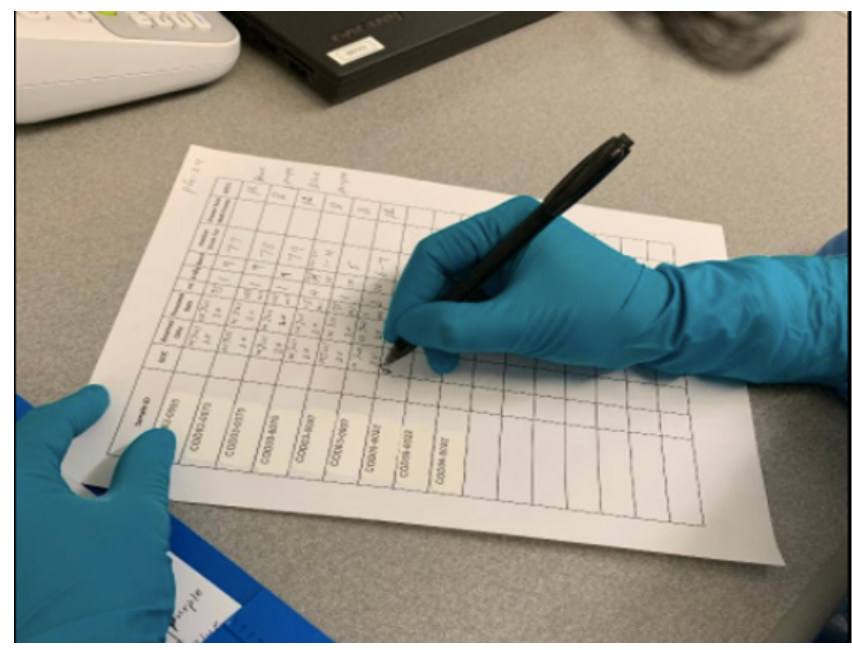

Figure 9. Paper log containing: subject ID, date received, volume $(\mathrm{mL})$, biobank box location \#, tube location, drawer/rack/shelf/freezer, comments.

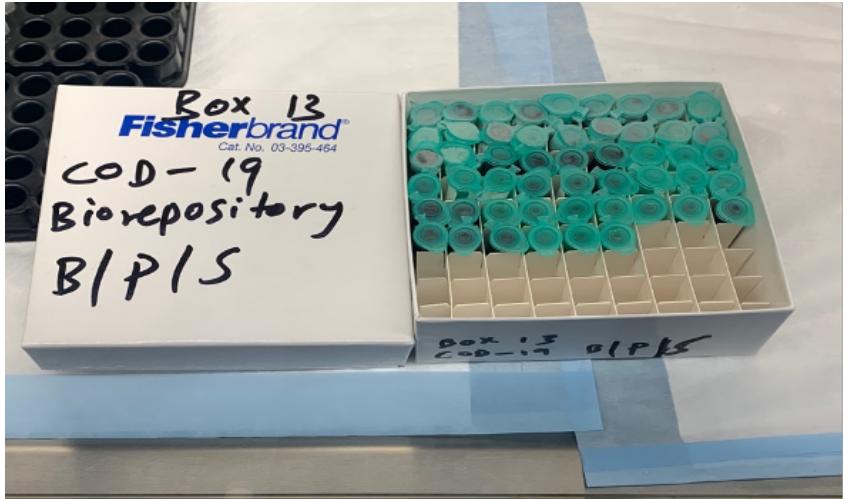

Figure 10. CRYO tubes placed in storage box. ferred the technician then capped and placed the tube on the completed tube rack. The technician continued this process until all the plasma collection tubes from the current day had been transferred into the CRYOtubes and placed on completed rack.

\section{Serum samples}

Laboratory technicians began the process by retrieving the sample tube from the biohazard bag and confirming the waste sample as serum. This identification was done by inspecting the label on the collection tube for a clear serum sample designation, or referring to the laminated informational guide for identifying sample types based on the lid color of the sample collection tube, or inspecting the contents of the tube to confirm if there is a distinctly separated serum layer in the tube. The technician then aliquoted $0.5 \mathrm{~mL}$ of the serum sample off the supernatant layer of the solution in the tube while making sure not to collect any volume from the WBC layer in the collection tube. Once collected the technician pipetted it into a CRYO tube then capped and placed on the completed tube rack. The technician continued this process until all the serum collection tubes from the current day had been transferred into the CRYO tubes and placed on completed rack.

\section{Cataloging the samples during laboratory processing}

After laboratory personnel have completed the initial COVID-19 sample transferring processing steps; the second step of the process was creating the biobank inventory log paper (Figure 8). This document contains subject identifiers, sample collection data, number of aliquots, volume aliquoted, sample type and storage locations that the tube will occupy after the biobank pro- cessing is completed. This highlights one of the most important steps of the data collection process as it allows the COVID-19 sample to be tracked over its storage life (five-year maximum) in the biobank in addition to be the source document for all future data entry related to the samples. This data collection process was performed by two laboratory technicians to ensure quality assured data accuracy and safe laboratory techniques when working with COVID-19 samples.

While laboratory personnel continue to work in the BSC, the second laboratory technician works in parallel to document and produce the biobank inventory log. During this process one technician is designated to work under BSC, transcribing the collection samples one at a time and verbally documenting and verifying the requested sample log information to the other laboratory technician. As the technician obtained the data, they record it onto the biobank inventory paper $\log$ (Figure 9). When all information is retrieved off of the collection sample tubes the samples are stored in freezer boxes (Figure 10). The technician then moved forward with the data management work by beginning to complete the log and then transferring the sample data to the electronic version of the log.

\section{Transfer of the sample log data}

Once the paper copy of the biobank inventory log contained the data from the hospital collection tubes the focus of the technician was to transfer the data from the inventory log sheet to the laboratory data excel file (Figure 11). The data are transferred to the electronic version by technicians for two purposes. Maintaining this log electronically on an encrypted university machine adds another layer of security for sensitive data 


\begin{tabular}{|c|c|c|c|c|c|c|c|c|}
\hline $\begin{array}{c}\text { Record } \\
\text { ID }\end{array}$ & $\begin{array}{c}\text { Subject } \\
\text { ID }\end{array}$ & $\begin{array}{c}\text { Received } \\
\text { Date }\end{array}$ & $\begin{array}{c}\text { Processed } \\
\text { date }\end{array}$ & $\begin{array}{c}\text { Volume } \\
\text { (mL) }\end{array}$ & $\begin{array}{c}\text { Number of } \\
\text { aliquots }\end{array}$ & $\begin{array}{c}\text { Biobank box } \\
\text { location } \\
\text { number }\end{array}$ & $\begin{array}{c}\text { Tube } \\
\text { location } \\
\text { (from- } \\
\text { to) }\end{array}$ & $\begin{array}{c}\text { Drawer } \\
\text { Rack } \\
\text { Shelf } \\
\text { Freezer }\end{array}$ \\
\hline & & & & & & & & \\
\hline & & & & & & & & \\
\hline & & & & & & & & \\
\hline & & & & & & & & \\
\hline
\end{tabular}

Figure 11. Biobank Excel inventory log.

and it allows multiple teams at various locations in the division to access the data as needed. This is important as specific sensitive biobank data may be required by personnel throughout CERID for tasks such as; data entry into the REDCap data base, quality checks for all steps of the data management process, biobank inventory inquiries, and miscellaneous research tasks.

\section{Application of the sample identification labels}

As the transfer of the daily sample delivery into the CRYO tubes was completed, the two technicians turned their attention to the last process step prior to the samples being stored in the biobank. This step involved the application of the CRYO tube identification labels to the CRYO tubes and the recording of the subject ID number from the CRYO label onto the laboratory logs under the correct research sample. In this process technician one removes the CRYO label from the biohazard bag and matches it to the correct CRYOtube on the completed sample rack and applies it, so the all digits are visible. Laboratory technician then communicates the unique sample ID to technician two to record it on both the hard copy log and in the electronic version of the log. The process of applying labels and recording sample ID's continues until all samples had been labeled and recorded.

\section{Storage of samples in the biobank}

After the sample processing has been completed the CRYO tubes are ready to be placed in the biobank for storage. Storage of the samples must align with the daily biobank location within the CRYO box in which it will be stored. Each box has space dividers, enabling the box to hold 81 CRYO tubes. Thus, each sample will then have a spot designated between 1-81. Each box itself will also be numbered due to the frequency, volume, and complexity of samples for the study and its associated subjects. The box is then given a permanent location within a -80 degrees $C$ Freezer. Due to the number of specimens housed in the UL Biobank, multiple freezers are needed, and each freezer is numbered. Within each freezer is a numbered shelf (1-4), rack (1-5), drawer (A-D), and position (A1-D4) (Figure 12). This detailed arrangement makes for a comprehensive and easily locatable biobank of biological specimens, making it efficient, convenient, and quality assured.

\section{Biobank sample request process development}

The CERID leadership's main objective of creating a COVID-19 biobank was to build an abundant repository of various samples from patients testing positive for SARS-CoV-2 and reducing any barriers to obtaining samples in support of research. To achieve this goal CERID leadership and the laboratory unit agreed that anyone interested in biobank samples should simply contact the CERID director to make the request. The CERID director would then facilitate the transfer of the sample(s). The biobank team will expedite shipment of the requested samples and update the inventory of available aliquots.

\section{Quality measures}

The CERID Quality Assurance Unit is responsible for ensuring research accuracy at every stage of the process. CERID leadership understood that the work of identifying and correcting the errors that arose from such an intensive process was critical in maintaining the integrity of the biobank and in providing quality data to future researchers. This process was accomplished by assigning specific researchers from the quality unit to conduct a systematic evaluation of the process. These personnel were trained in each step of the process and helped in the construction of the quality metrics. These metrics and the quality steps utilized in the process can be seen in the following table (Table 1).

\section{Discussion}

The novel SARS-CoV-2 virus rapidly spread across the globe causing increased morbidity and mortality. During a pandemic, recognition of acquiring waste samples from persons infected with a new disease is essential for continued research projects. Waste samples are necessary for understanding the viral properties, mutations, development of treatment modalities, development of successful medication regimens, and vaccine 
Table 1. Quality metrics for development of the COVID-19 biobank.

Quality Metrics for the development of a COVID-19 biobank

Process Steps

Quality Metric

1) Specimen database development and maintenance.

HIPAA and IRB compliant.

Restricted gatekeeping ability for section entry based on the need and task related to specific team members.

Realtime structure editing based on continuous program evaluation

2) Production of the sample identification code.

Unique deidentified alpha numeric code containing study name, sample collection location, and subject

3) Specimen collection and transport from hospitals to the laboratory.

Packaging sample appropriately for transport CRYO labels accompanying each specimen

Specimen biohazard bag labelled

Specimen label legible

4) Data entry and generation of REDCap ID number.

Mandatory data completion fields to prevent missing data

Utilization of check boxes and drop-down choices to prevent text data errors

Certification of all demographic information

Specimen type verified verses lab record

Identity of data entry personnel via history feature

5) Processing of the samples for placement in biobank.

Mandatory PPE for staff in contact with positive samples.

6) Cataloging the samples during laboratory processing.

7) Application of the sample identification labels.

Protocol requiring two lab members (crosscheck) working together to prevent error in all lab steps of the process

8) Storage of samples in the biobank.

9) Biobank sample request process development.

10) Quality measures. 


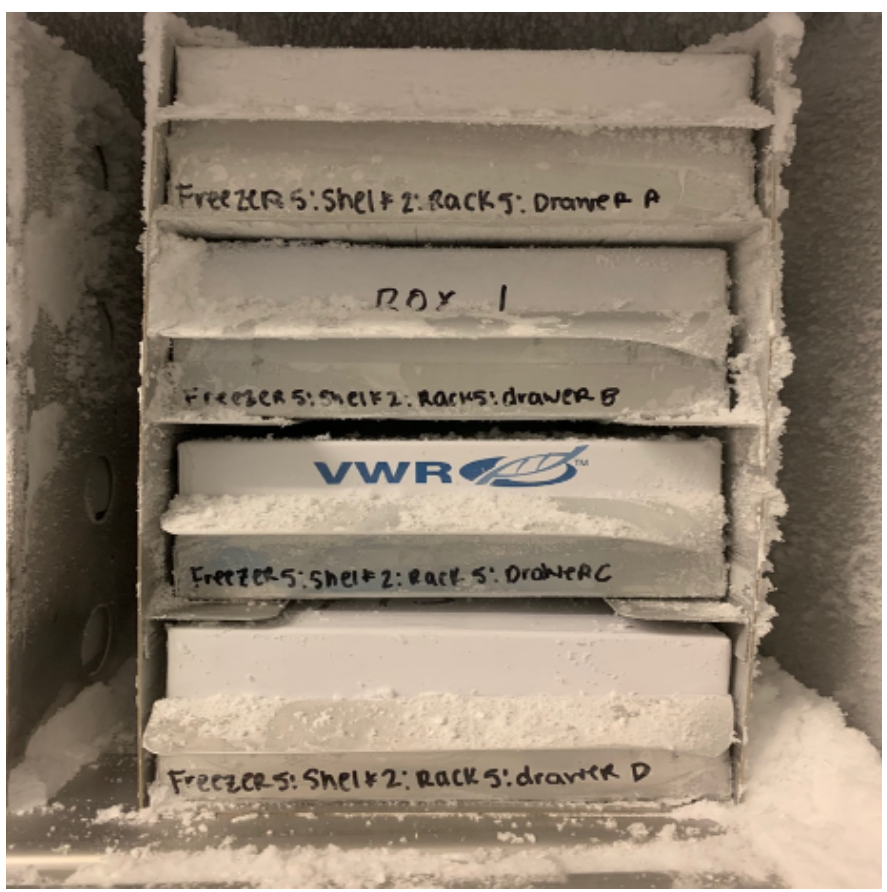

Figure 12. Freezer placement.

development to name a few. The hope is that the information provided in this methodologies article provides guidance for others considering creating a new or adding to an existing biobank to provide researchers specimens to help prevent morbidity and mortality in this and other pandemics in the future.
Received: August 27, 2020

Accepted: December 2, 2020

Published: December 7, 2020

Copyright: (c) 2022 The author(s). This original article is brought to you for free and open access by ThinkIR: The University of Louisville's Institutional Repository. For more information, please contact thinkir@louisville.edu. This article is distributed under the terms of the Creative Commons Attribution 4.0 International License (CC BY 4.0), which permits unrestricted use, distribution, and reproduction in any medium, provided the original author and source are credited.

Funding Source: The author(s) received no specific funding for this work.

Conflict of Interest: All authors declared no conflict of interest in relation to the main objective of this work.

\section{References}

1. Ramirez JA, Palmer KE, Carrico R, Arnold FW, Chung D, Wolf LA. Louisville Coronavirus Surveillance Program. Univ Louisville J Respir Infect 2020; 4(1):Article 3. doi: 10.18297/rgh/vol4/iss $1 / 3$.

2. Carrico $R$, Balcom $D$, Johnson $W$, et al. Implementation of the Louisville COVID-19 Surveillance Protocol: Experiences from the University of Louisville Center of Excellence for Research in Infectious Diseases (CERID). Univ Louisville J Respir Infect 2020; 4(1):Article 10. doi: $10.18297 / \mathrm{rgh} / \mathrm{vol} 4 / \mathrm{iss} 1 / 10$.
3. De Souza YG, Greenspan JS. Biobanking past, present and future: Responsibilities and benefits. AIDS 2013; 27(3):303-12. doi: 10.1097/QAD.0b013e32835c1244. PMID: 23135167.

4. Harris PA, Taylor R, Thielke R, Payne J, Gonzalez N, Conde JG. Research electronic data capture (REDCap)-a metadata-driven methodology and workflow process for providing translational research informatics support. J Biomed Inform 2009; 42(2):377-81. doi: 10.1016/j.jbi.2008.08.010. PMID: 18929686. 